disadvantage. A limitation of this study is that SEC measures are not directly comparable across countries.

\section{OP104 DISTINCT PATTERNS OF SOCIO-ECONOMIC DISPARITIES IN CHILD-TO-ADOLESCENT BMI TRAJECTORIES ACROSS UK ETHNIC GROUPS: A PROSPECTIVE LONGITUDINAL STUDY}

${ }^{1} Y$ Lu*, ${ }^{2} \mathrm{~A}$ Pearce, ${ }^{1} \mathrm{~L} \mathrm{Li} .{ }^{\prime}$ Great Ormond Street Institute of Child Health, University College London, London, UK; ${ }^{2}$ MRC/CSO Social and Public Health Sciences Unit, University of Glasgow, Glasgow, UK

\subsection{6/jech-2019-SSMabstracts. 107}

Background In many high-income countries, BMI and levels of overweight and obesity are inversely associated with socio-economic status. Recent evidence suggests that socio-economic disparities in BMI are emerging at a young age. Little is known whether patterns of these disparities vary by ethnicity, especially in the UK. This is the first UK study to our knowledge to examine the pattern of socio-economic disparities in child-to-adolescent BMI trajectories across ethnic groups.

Methods We used data from the UK Millennium Cohort Study, which oversampled children living in the disadvantaged circumstances and in England those from minority ethnic backgrounds. A total of 15,996 children with 62,051 BMI measurements between 3 and 14 years were included in this analysis. Mixed-effects fractional polynomial models were applied to estimate mean BMI trajectories for each socio-economic group (as defined by poverty and maternal education) and differences in BMI between groups at each age. Models were subsequently stratified by ethnicity and adjusted for maternal pre-pregnancy BMI, maternal smoking during pregnancy, birthweight and infant feeding.

Results Overall,the poverty group had a higher mean BMI than non-poverty group from 6 years with a small difference of $0.06 \mathrm{~kg} / \mathrm{m}^{2}$ [95\% CI $\left.0.01-0.12\right]$, which increased to 0.67 $\mathrm{kg} / \mathrm{m}^{2}[0.52-0.82]$ by 14 years. The income-BMI associations differed by ethnicity. In Whites and South Asians, the BMI difference by income was established at 3 years and widened with age. Among Black African-Caribbeans, there was a reverse socio-economic gradient in $\mathrm{BMI}$, in that the poverty group had a lower BMI $\left(-0.37 \mathrm{~kg} / \mathrm{m}^{2}[-0.04,-0.71]\right.$ at 5 years; $-0.95 \mathrm{~kg} / \mathrm{m}^{2}[-0.11,-1.79]$ at 14 years). Differences remained after adjustment for early-life factors. These distinct patterns persisted when using maternal education as the socioeconomic indicator.

Conclusion These findings imply that socio-economic advantage may not necessarily be universally associated with lower BMI. The effect of socio-economic circumstances on BMI potentially differs by ethnic group. Given the increasing ethnic diversity in the UK, public health approaches to promote healthy weight need to consider the varying needs of target populations. The positive income-BMI association found in Black African-Caribbean children requires replication in other samples and further investigation into the underpinning cultural and biological mechanisms that may explain these differences.

\section{OP105 ASSOCIATIONS BETWEEN NEIGHBOURHOOD ENVIRONMENTS AND HOSPITAL ADMISSIONS FOR CVD ARE MODIFIED BY SOCIOECONOMIC FACTORS: A PROSPECTIVE STUDY USING UK BIOBANK}

${ }^{1,2}{ }^{2}$ E Mason* ${ }^{*}{ }^{1} \mathrm{~N}$ Pearce, ${ }^{3} \mathrm{~S}$ Cummins. ${ }^{1}$ Epidemiology and Population Health, London School of Hygiene and Tropical Medicine, London, UK; ${ }^{2}$ Public Health and Policy, University of Liverpool, Liverpool, UK; ${ }^{3}$ Public Health, Environments and Society, London School of Hygiene and Tropical Medicine, London, UK

\subsection{6/jech-2019-SSMabstracts. 108}

Background Neighbourhood environments may influence risk of cardiovascular disease (CVD), via diet and physical activity (PA) behaviours. However, if the effects of CVD-related neighbourhood risks vary by socioeconomic position, efforts to improve population health by improving neighbourhood built environments may widen health inequalities. We examined whether associations between two neighbourhood characteristics - availability of PA facilities and fast-food store proximity - and CVD-related hospital admissions, were modified by income and area deprivation.

Methods 336,156 UK Biobank participants aged 40-70 years, linked to the UK Biobank Urban Morphometric Platform, were followed up through linked Hospital Episodes Statistics (mean follow-up $=6.8$ years). We examined whether associations between neighbourhood density of formal PA facilities and proximity of home address to a fast-food/takeaway store (at baseline), and hospital admissions with a primary diagnosis of CVD, were modified by household income or area deprivation (Townsend). We used Cox proportional hazards models, adjusted for likely confounding, and calculated relative excess risks due to interaction (RERI) to assess effect modification on the additive scale. We also examined the combined modifying role of income and deprivation.

Results Household income and area deprivation modified associations between neighbourhood exposures and CVD-related hospital admissions. Greater density of PA facilities may have a larger public health impact in more deprived areas $($ RERI $=0.088)$, but high-income households benefit more than low-income households (RERI=-0.075). The estimated benefit was restricted to high-income households in deprived areas, where we observed 21\% lower hazard of being admitted to hospital with CVD for people living $<1 \mathrm{~km}$ from at least four PA facilities than among people with no local PA facilities $(\mathrm{HR}=0.79$, 95\%CI:0.65-0.95). For fast-food proximity, reduced access to fast-food stores might have the biggest impact for low-income households (RERI $=0.075)$, but mostly in less deprived areas (RERI=-0.104). A beneficial association was only observed among low-income households in affluent areas, where the hazard of CVD-related admission was $12 \%$ lower among people living $\geq 2 \mathrm{~km}$ from a fast-food store than among people living $<500 \mathrm{~m}$ from one $(\mathrm{HR}=0.88,95 \%$ CI:0.80-0.97).

Conclusion Among mid-life adults in the UK, associations between neighbourhood food and PA environments and hospital admissions for CVD varied according to household income and area deprivation. Results suggest that formal PA facilities may reduce CVD risk in deprived areas, but not among lowincome households, raising important implications for health 